\title{
Multicentre European study for the treatment of advanced emphysema with bronchial valves
}

\author{
Vincent Ninane*, Christian Geltner", Michela Bezzi", Pierfranco Foccoli", \\ Jens Gottlieb ${ }^{+}$, Tobias Welte ${ }^{+}$, Luis Seijo ${ }^{\S}$, Javier J. Zulueta ${ }^{\S}$, Mohammed Munavvar ${ }^{f}$, \\ Antoni Rosell**, Marta Lopez**, Paul W. Jones ${ }^{\# \#}$, Harvey O. Coxson ${ }^{\star \tau}$, \\ Steven C. Springmeyer ${ }^{++}$and Xavier Gonzalez ${ }^{++}$
}

ABSTRACT: This multicentre, blinded, sham-controlled study was performed to assess the safety and effectiveness of bronchial valve therapy using a bilateral upper lobe treatment approach without the goal of lobar atelectasis.

Patients with upper lobe predominant severe emphysema were randomised to bronchoscopy with $(n=37)$ or without $(n=36)$ IBV Valves for a 3-month blinded phase. A positive responder was defined as having both $a \geqslant 4$-point improvement in St George's Respiratory Questionnaire (SGRQ) and a lobar volume shift as measured by quantitative computed tomography.

At 3 months, there were eight (24\%) positive responders in the treated group versus none $(0 \%)$ in the control group $(p=0.002)$. Also, there was a significant shift in volume in the treated group from the upper lobes (mean \pm SD $-7.3 \pm 9.0 \%$ ) to the non-treated lobes $(6.7 \pm 14.5 \%)$, with minimal change in the control group $(p<0.05)$. Mean SGRQ total score improved in both groups (treatment: $-4.3 \pm 16.2$; control: $-3.6 \pm 10.7$ ). The procedure and devices were well tolerated and there were no differences in adverse events reported in the treatment and control groups.

Treatment with bronchial valves without complete lobar occlusion in both upper lobes was safe, but not effective in the majority of patients.

KEYWORDS: Bronchial valve therapy, emphysema, quantitative computed tomography, St George's Respiratory Questionnaire

hronic obstructive pulmonary disease (COPD) is a progressive, debilitating, chronic respiratory disease that results in significant morbidity and mortality [1]. Emphysema, characterised by parenchymal destruction, affects an estimated $1.8 \%$ of the population worldwide [2]. Although there has been a rapid expansion in our understanding of COPD pathogenesis over the past $20 \mathrm{yrs}$, there is a marked scarcity of treatments that can modify disease progression and reduce mortality. Pharmacological therapies can be effective on airway obstruction, but their actions are more limited in the emphysema phenotype, in which the main mechanism of airflow limitation is the loss of elastic recoil associated with air trapping and hyperinflation $[3,4]$. Therefore, for patients with advanced emphysema who have maximised their medical treatment, few options are available to manage their severe symptoms.
During the 1990s, treatment of severe emphysema turned to surgical procedures such as lung volume reduction surgery (LVRS). Subsequently, the National Emphysema Treatment Trial (NETT) compared the effects of LVRS with medical therapy and showed significant survival benefit in the LVRS group [5], in addition to the improved quality of life, physiology and exercise capacity previously shown $[6,7]$. However, due to the risks associated with LVRS, the majority of patients are ineligible [6] or unwilling to undergo LVRS, and no more than 300 procedures are estimated to be performed annually in the USA [8].

Bronchoscopic methods to treat emphysema with one-way bronchial valves have been developed in order to achieve benefits similar to those of LVRS, without the associated risks [9, 10]. Early data with bilateral bronchial valve treatment
AFFILIATIONS

${ }^{*} \mathrm{CHU}$ St Pierre Hospital, Brussels, Belgium.

\# Landeskrankenhaus Natters,

Innsbruck, Austria.

'Spedali Civili di Brescia, Brescia, Italy.

+Medizinische Hochschule,

Hannover, Germany

${ }^{\S}$ Clinica Universidad de Navarra, Pamplona,

**Hospital Universitari de Bellvitge, Barcelona, Spain.

${ }^{f}$ Royal Preston Hospital, Preston,

\#\# Division of Clinical Science, St

George's University of London,

London, UK.

"Dept of Radiology, Vancouver

General Hospital, Vancouver, BC,

Canada.

+'Spiration Inc., Redmond, WA, USA

CORRESPONDENCE

$\checkmark$. Ninane

CHU St Pierre Hospital

Rue Haute 322

1000 Brussels

Belgium

E-mail: Vincent NINANE@stpierre-

bru.be

Received:

Feb 032011

Accepted after revision:

March 202012 


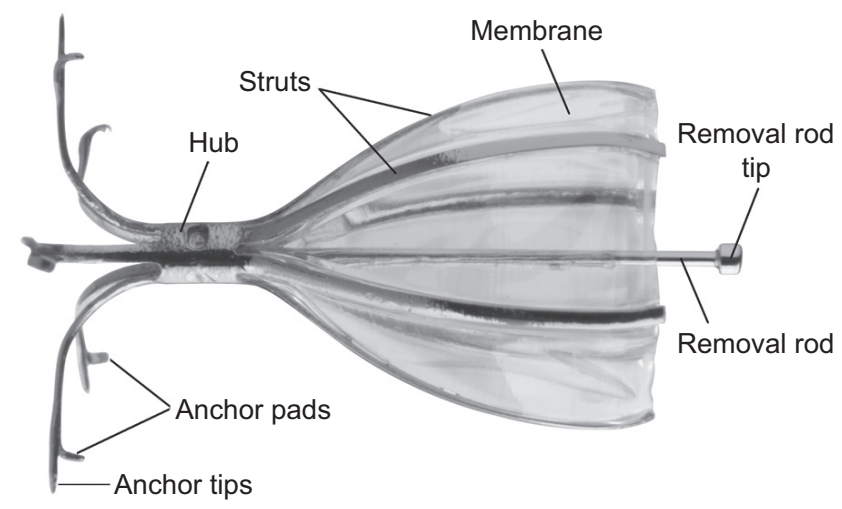

FIGURE 1. Diagram of the IBV one-way valve (Spiration Inc., Redmond, WA, USA), consisting of a nitinol frame covered with a polymer membrane with six struts and five anchors that securely engage the airway walls at the targeted treatment location.

showed clinical improvement in patients with and without the presence of lobar atelectasis [11, 12]. Recent data shows the potential survival benefit when single-lobe lobar atelectasis is produced [13]. It has been found that the treatment effects from bronchial valve therapy are the result of contributions of at least three mechanisms of action: lobar shift of ventilation and perfusion from treated to untreated lung regions [14]; reduction of dynamic hyperinflation [11]; and volume reduction [9].

The IBV Valve (Spiration Inc., Redmond, WA, USA; fig. 1) has been successfully used in 91 patients in a pilot study demonstrating clinical improvements in both lung volumes and health status outcomes [15]. In this pilot study, an association between lobar atelectasis and pneumothorax, and deaths related to pneumothorax was described. The European study reported here is the first blinded, controlled evaluation of any bronchial valve therapy in advanced emphysema. Response to treatment was based on a composite end-point of health status as measured by the St George's Respiratory Questionnaire (SGRQ) [16] and regional lung volume changes as measured by quantitative computed tomography (CT) [14].

\section{METHODS}

This was a prospective, randomised, multicentre, singleblinded, sham-controlled study (clinicaltrials.gov identifier NCT00880724). Eligible subjects underwent bronchoscopy and valve placement (treatment group) or a sham bronchoscopy (control group) and were followed for 3 months. At 3 months, treatment assignment was un-blinded and subjects on valve therapy were evaluated for a further 3 months. Eligible subjects in the control group could then receive bronchial valves in an un-blinded fashion and were followed-up for 3 months. Subject flow through the study is shown in figure 2 .

Patients with advanced upper lobe predominant emphysema underwent medical evaluation to ensure compliance with treatment and eligibility for the inclusion and exclusion criteria that are detailed in the online supplementary material. Pulmonary rehabilitation was not a prerequisite. The study was approved by each ethics committee according to local laws and regulations. Written informed consent was obtained from each subject.

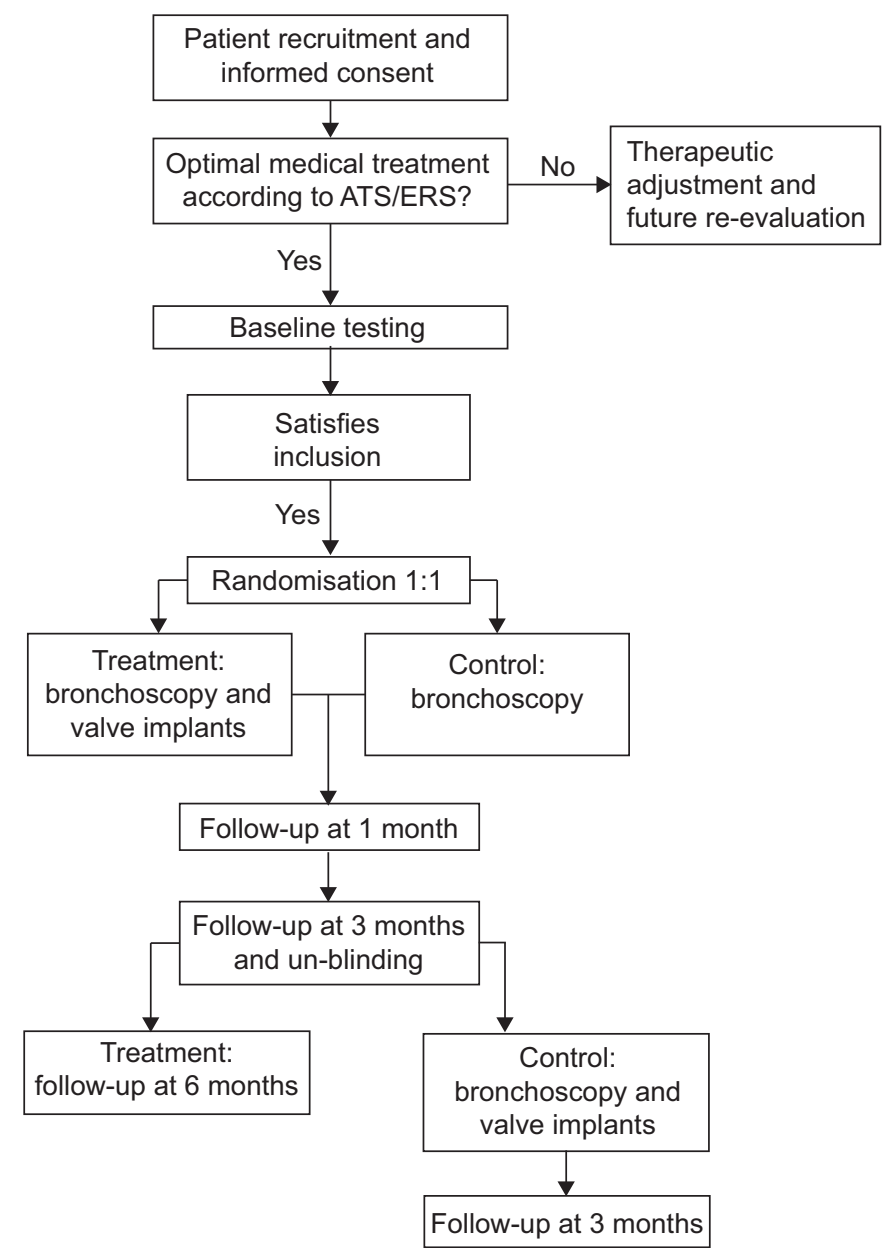

FIGURE 2. Subject flow through the study. ATS: American Thoracic Society; ERS: European Respiratory Society.

\section{Bronchial valve and procedure}

The IBV Valve is an umbrella-shaped, one-way valve that limits airflow into targeted airways distal to the valve but allows mucus and air movement in the proximal direction and, if needed, is removable (fig. 1).

Airways in the upper lobes were measured using a calibrated balloon catheter to determine appropriate valve size $(5,6$ or $7 \mathrm{~mm}$ ). Valves were then placed in the airways by catheter delivery through a flexible bronchoscope. Per protocol, one segment or sub-segment of the right upper lobe and the lingula segments of the left upper lobe were not treated to achieve incomplete occlusion of the upper lobes and to prevent lobar atelectasis. The un-treated right upper lobe segment was determined by the investigator based upon CT emphysema distribution evaluation.

Randomisation occurred after deep sedation and/or general anaesthesia and bronchoscopic examination of the airways. Subjects were discharged the day after the procedure and scheduled for evaluation 1 and 3 months later.

\section{Outcome measures}

The study outcome measure was based on a composite endpoint of the SGRQ total score (responder defined as change of 


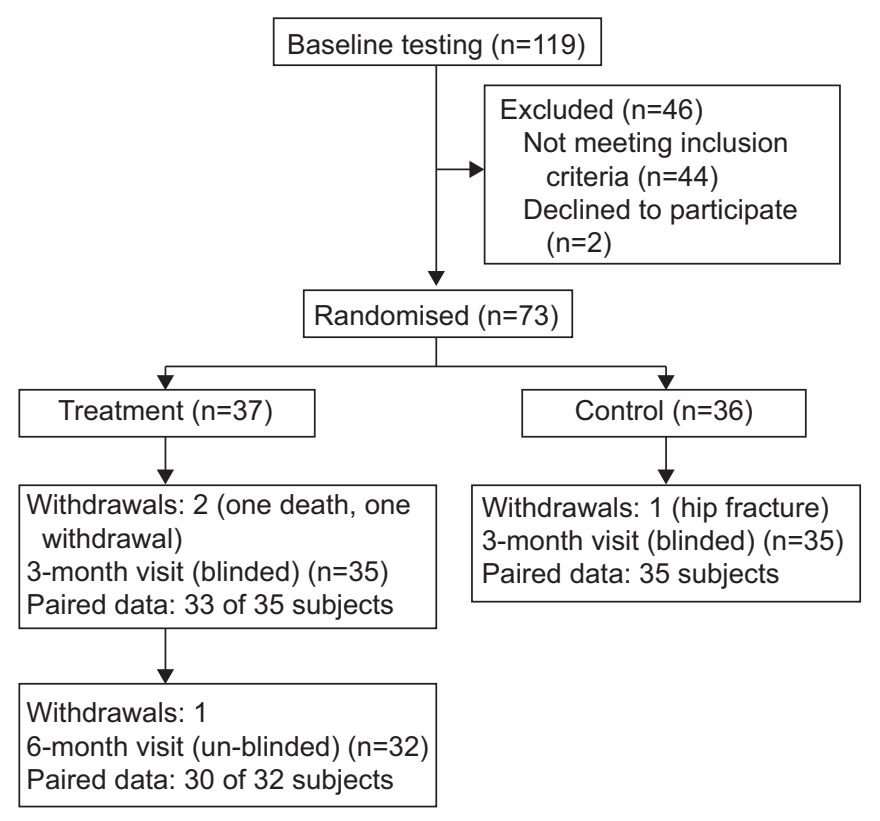

FIGURE 3. Subject distribution during the study.

$\geqslant 4$ points) [17] and lung volume changes measured by CT scans (volume decrease in upper lobes with a compensatory volume increase in non-treated lobes of $\geqslant 7.5 \%$ ). This threshold for the non-upper lobe increase was revised from pilot data where a $10 \%$ threshold was used [14], based on a distributive method. Since the present study had a control group, it was considered that if the volumetric threshold was set too high, there was a risk that patients in the control group having volumetric changes and/or variations due to improved clinical management, placebo effect or pathological conditions (for example pneumonia), would be inappropriately deemed non-responders.

Other evaluations included: health status measures (Short Form health survey-36 [18] and modified Medical Research Council dyspnoea scale [19]), pulmonary function tests (forced expiratory volume in $1 \mathrm{~s}$ (FEV1), total lung capacity and residual volume), diffusing capacity of the lung for carbon monoxide, 6-min walking test (6MWT) and arterial blood gases. Adverse events were monitored throughout the study and were classified as anticipated or unanticipated. Each adverse event was reported by the investigator according to severity (serious, severe, moderate or mild) and relationship with the devices (definitely, probably, possibly and not device-related).

\section{Imaging}

CT scans were obtained using multidetector row scanners at full suspended inspiration and with standardised parameters (details given in online supplementary material).

Quantitative analysis of lung volumes was performed at the University of British Columbia, Vancouver, BC, Canada using Pulmonary Workstation software version 2.0 (VIDA Diagnostics, Iowa City, IA, USA). Information on the analysis is detailed in the online depository.

\section{Statistical analysis}

There was no formal power calculation for this study but the sample size of 100 patients was selected in order to estimate the difference in responder rates with a maximum standard error of $10 \%$. After a 2-yr study period, enrolment was stopped for logistical reasons, at which point 73 patients had been enrolled.

Continuous measures were compared between groups via t-tests, both paired and unpaired as required. Comparisons of count data (such as number of primary outcome responders or adverse events) were made using Fisher's exact test. All tests were twosided. A p-value $<0.05$ was considered statistically significant.

\section{RESULTS}

\section{Study population and clinical characteristics}

73 subjects were recruited to the study in seven sites across six countries, of whom 37 received valve treatment and 36 were in the control group (fig. 3). Clinical characteristics at baseline are presented in table 1; the two groups were well matched in terms of demography, health status and physiological assessments.

\section{Bronchoscopic procedure}

The mean \pm SD number of valves placed was $7.3 \pm 2$ in the treatment group. The mean \pm SD procedure time in the treatment group was $62 \pm 17 \mathrm{~min}$, approximately double of that in the control group, due to the valve placement procedure (table 2). There was no difference in the amount of time spent in hospital or type of adverse events associated with the procedure between study groups, all of which were anticipated events and of a low incidence (table 2).

\section{Outcome measure}

The study outcome measure showed a highly significant difference between treatment and control groups. Using paired data (subjects with both CT and SGRQ results at baseline and follow-up period), eight $(24 \%)$ out of 33 subjects in the

\begin{tabular}{|c|c|c|c|}
\hline \multicolumn{4}{|c|}{$\begin{array}{l}\text { Baseline demographic and clinical } \\
\text { characteristics }\end{array}$} \\
\hline Characteristic & Treatment & Control & p-value \\
\hline Subjects $n$ & 37 & 36 & \\
\hline Age yrs & $61 \pm 7$ & $62 \pm 6$ & 0.363 \\
\hline Males n (\%) & $23(62)$ & $20(56)$ & 0.683 \\
\hline FEV $1 \%$ pred & $35 \pm 10$ & $32 \pm 7$ & 0.122 \\
\hline TLC \% pred & $130 \pm 19$ & $136 \pm 18$ & 0.146 \\
\hline RV \% pred & $238 \pm 74$ & $258 \pm 67$ & 0.230 \\
\hline SGRQ total score & $61 \pm 11$ & $60 \pm 13$ & 0.766 \\
\hline MMRC dyspnoea score & $2.8 \pm 0.7$ & $2.8 \pm 0.9$ & 0.869 \\
\hline 6MWT m & $337 \pm 106$ & $346 \pm 123$ & 0.726 \\
\hline \multicolumn{4}{|c|}{ Arterial blood gases $\mathrm{mmHg}$} \\
\hline $\mathrm{PO}_{2}$ & $65 \pm 10$ & $66 \pm 10$ & 0.879 \\
\hline $\mathrm{PCO}_{2}$ & $40 \pm 5$ & $40 \pm 4$ & 0.486 \\
\hline \multicolumn{4}{|c|}{$\begin{array}{l}\text { Data are presented as mean } \pm \mathrm{SD} \text {, unless otherwise stated. FEV1: forced } \\
\text { expiratory volume in } 1 \mathrm{~s} \text {; \% pred: \% predicted; TLC: total lung capacity; RV: } \\
\text { residual volume; SGRQ: St George's Respiratory Questionnaire; MMRC: } \\
\text { modified Medical Research Council; } 6 \mathrm{MWT} \text { : 6-min walking test; } \mathrm{PO}_{2} \text { : oxygen } \\
\text { tension; } \mathrm{PCO}_{2} \text { : carbon dioxide tension. }\end{array}$} \\
\hline
\end{tabular}




\begin{tabular}{|c|c|c|c|}
\hline Characteristic & Treatment $^{\#}$ & Control" & p-value \\
\hline Procedure time $\mathrm{min}$ & $62 \pm 17$ & $23 \pm 14$ & $<0.0001$ \\
\hline Total valves implanted $n$ & 270 & NA & NA \\
\hline $\begin{array}{l}\text { Number of valves per } \\
\text { subject }\end{array}$ & $7.3 \pm 2$ & NA & NA \\
\hline Days hospitalised $^{+}$ & $1.1 \pm 0.3$ & $1.1 \pm 0.3$ & 0.26 \\
\hline \multicolumn{4}{|l|}{ Procedural adverse events ${ }^{s}$} \\
\hline ABGs/acidosis & 1 & 0 & 1 \\
\hline Bronchospasm & 2 & 2 & 1 \\
\hline COPD exacerbation & 0 & 1 & 0.493 \\
\hline Dyspnoea & 2 & 0 & 0.493 \\
\hline Hypoxaemia & 1 & 1 & 1 \\
\hline Nausea & 2 & 0 & 0.493 \\
\hline Any adverse event & 6 & 3 & 0.479 \\
\hline Any serious adverse event & 0 & 1 & 0.493 \\
\hline
\end{tabular}

Data are presented as mean $\pm \mathrm{SD}$, unless otherwise stated. NA: not applicable; ABGs: arterial blood gases; COPD: chronic obstructive pulmonary disease. \#: $n=37 ; \because: n=36 ;^{+}$: protocol required an overnight stay after the procedure; \$: subjects (n) with at least one episode.

treatment group exceeded the minimum threshold changes for CT lung volumes and SGRQ total score, compared with none out of 35 subjects in the control group $(p=0.002)$ (table 3 ). Using all data, the result was the same with eight $(23 \%)$ responders out of 35 subjects in the treatment group and none out of 35 subjects in the control group $(p=0.005)$.

Analysis of paired data showed that at the end of the 3-month blinded phase, bronchial valve treatment resulted in a significant decrease in upper lobe lung volumes, and a significant corresponding increase in non-treated lobe volumes (upper lobe: $-7.3 \pm 9 \% \quad(-251 \pm 277 \mathrm{~mL})$; non-upper lobe: $6.7 \pm 14.5 \%$ $(221 \pm 471 \mathrm{~mL}) ; \mathrm{n}=33)$ compared with the control group, in which minimal changes in lung volumes were observed

\begin{tabular}{|c|c|c|c|c|}
\hline \multirow[t]{2}{*}{ TABLE 3} & \multicolumn{4}{|c|}{$\begin{array}{l}\text { Per cent responders and change from baseline } \\
\text { in computed tomography (CT) lung volumes and } \\
\text { St George's Respiratory Questionnaire (SGRQ) } \\
\text { score at } 3 \text { months, and per cent of responders } \\
\text { (paired data) }\end{array}$} \\
\hline & & Treatment & Control & p-value \\
\hline \multirow{2}{*}{\multicolumn{2}{|c|}{$\begin{array}{l}\text { CT volumes and SGRQ } \\
\text { responders" } n \\
\text { CT lung volumes } \% \text { change }\end{array}$}} & $8 / 33$ & $0 / 35$ & 0.002 \\
\hline & & & & \\
\hline \multicolumn{2}{|c|}{ Upper lobes (treated) } & $-7.3 \pm 9$ & $0.7 \pm 5.2$ & $<0.0001$ \\
\hline \multicolumn{2}{|c|}{ Non-upper lobes (untreated) } & $6.7 \pm 14.5$ & $0.2 \pm 7.8$ & 0.027 \\
\hline \multicolumn{2}{|c|}{ SGRQ total score } & $-4.3 \pm 16.2$ & $-3.6 \pm 10.7$ & 0.837 \\
\hline
\end{tabular}

(table 3). Similar shifts in lung volumes were also observed at 6 months in the treatment group (upper lobe: $-5.3 \pm 7.8 \%$; nonupper lobe: $7.8 \pm 9.3 \%$; $n=30$ ).

Both the treated and control groups showed an improvement in SGRQ scores from baseline (treatment: $-4.3 \pm 16.2$; control: $-3.6 \pm 10.7$ ) (table 3 ). Only the mean change in the treated group exceeded the minimal clinically important difference of 4 points, but for both groups there was a wide variation in mean scores, shown by the large standard deviations. A further improvement in SGRQ scores was shown at 6 months (mean change from baseline: $-10.9 \pm 18.2 ; \mathrm{n}=30$ ).

\section{Impact of COPD exacerbations on SGRQ total score}

Subjects who experienced COPD exacerbations during the 3-month blinded phase of the study had worsening SGRQ scores (mean change from baseline: $+3.1 \pm 10$ ) compared with a mean improvement in subjects without COPD exacerbations $(-5.2 \pm 14.7)$. However, there was no evidence that the effect of COPD flares was different for the treatment group versus the control group $(p=0.29)$.

\section{Other parameters evaluated}

A summary of other test results is shown in table 4 .

\section{Safety}

During the entire study, there were no reports of valve migration or erosion, and there was no expectoration of valves. After hospital discharge and during the 3-month blinded phase of the study, 24 (33\%) subjects reported one or more adverse events, 15 subjects in the treated group and nine subjects in the control group (table 5). There was no difference in the incidence of individual adverse events between the treated and control groups, and there were no reports of atelectasis and/or pneumothorax. All adverse events were classified as anticipated and no adverse events were assessed as definitely related to the valve therapy.

During the 3-month blinded period, one fatal event was reported for a subject that had a cerebrovascular accident 59 days after bronchial valve treatment, which was assessed as not related to the device. Beyond 3 months, two fatal events were reported: one subject died 138 days after valve treatment in the rollover group, which was assessed as unrelated to the device; another died from respiratory failure 241 days after valve treatment, which was assessed as possibly related to treatment.

\section{DISCUSSION}

This is the first report of a blinded, randomised and controlled study to evaluate the effects of bronchial valve therapy for the treatment of advanced emphysema. The bilateral upper lobe treatment approach used in this study was successful due to the highly acceptable safety outcomes coupled with the positive primary outcome. This study conclusion supports the earlier uncontrolled and non-blinded studies that suggested bronchial valve therapy of emphysema has acceptable safety and effectiveness [15, 20].

The composite primary outcome for this study was based on CT lung volumes and SGRQ total score, a meaningful improvement in both being required to be defined as a responder. This composite end-point reflects the clinical improvement resulting from a bilateral upper lobe treatment approach that produces 
TABLE 4 Other measures

\begin{tabular}{|c|c|c|c|c|c|}
\hline & \multicolumn{2}{|c|}{ Treatment } & \multicolumn{2}{|c|}{ Control } & \multirow[t]{2}{*}{ p-value $\#$} \\
\hline & Baseline & 3 months & Baseline & 3 months & \\
\hline FEV 1 L & $0.99 \pm 0.35(37)$ & $0.90 \pm 0.34(34)$ & $0.88 \pm 0.29(36)$ & $0.87 \pm 0.34$ & 0.065 \\
\hline TLC L & $7.38 \pm 1.30(37)$ & $7.45 \pm 1.32(34)$ & $7.80 \pm 1.52(36)$ & $7.65 \pm 1.50(35)$ & 0.055 \\
\hline RV L & $4.65 \pm 1.30(37)$ & $4.86 \pm 1.35(34)$ & $5.26 \pm 1.18(36)$ & $5.05 \pm 1.19(35)$ & 0.012 \\
\hline MMRC dyspnoea score & $2.8 \pm 0.7(37)$ & $2.5 \pm 1.0(35)$ & $2.8 \pm 0.9(36)$ & $2.7 \pm 0.9(35)$ & 0.641 \\
\hline SF-36 MCS & $41 \pm 12(37)$ & $41 \pm 13(35)$ & $41 \pm 10(36)$ & $42 \pm 11(35)$ & 0.83 \\
\hline SF-36 PCS & $33 \pm 7(37)$ & $33 \pm 8(35)$ & $34 \pm 7(36)$ & $34 \pm 8(35)$ & 0.73 \\
\hline 6MWT m & $337 \pm 106(37)$ & $344 \pm 118(33)$ & $346 \pm 123(36)$ & $353 \pm 131(34)$ & 0.410 \\
\hline \multicolumn{6}{|c|}{ Arterial blood gases $\mathrm{mmHg}$} \\
\hline $\mathrm{PO}_{2}$ & $65 \pm 10(37)$ & $69 \pm 12(34)$ & $66 \pm 10(36)$ & $64 \pm 10(34)$ & 0.045 \\
\hline
\end{tabular}

Data are presented as mean \pm SD (n), unless otherwise stated. FEV1: forced expiratory volume in $1 \mathrm{~s}$; TLC: total lung capacity; RV: residual volume; DL,CO: diffusing capacity of the lung for carbon monoxide; MMRC: modified Medical Research Council; SF-36: Short Form health survey; MCS: mental component summary; PCS: physical component summary; 6MWT: 6-min walking test; $\mathrm{PO}_{2}$ : oxygen tension; $\mathrm{PCO}_{2}$ : carbon dioxide tension. ${ }^{\#}$ : change from baseline, treatment versus control.

volume shifts without lobar atelectasis. Composite end-points are widely used in clinical studies, especially for medical devices, and for this study, we selected a novel measurement of lung volume change coupled with a validated quality of life instrument (SGRQ) with specified thresholds.

Earlier studies with bronchial valves aimed to emulate the effects of LVRS by inducing lobar atelectasis, using complete occlusion of all airways in a single lobe $[9,10,21]$. However, in these studies, lobar atelectasis was not produced consistently, and when achieved, it was associated with a greater incidence of pneumothorax, most probably due to limited inter-lobar collateral ventilation. The results of the Endobronchial Valve for Emphysema Palliation Trial (VENT) demonstrated modest improvements in FEV1 and exercise tolerance but an accompanying worsening rate of a composite complications endpoint [21]. Consequently, the advantages and disadvantages of

\section{TABLE 5 All adverse events during the 3-month blinded} study period

\begin{tabular}{lccc} 
Adverse event & Treatment & Control & p-value \\
\hline Any adverse event & $15(20)$ & $9(10)$ & 0.214 \\
Any serious adverse event & $7(8)$ & $4(4)$ & 0.515 \\
COPD exacerbation & $11(13)$ & $8(9)$ & 0.595 \\
Bronchospasm & $1(1)$ & 0 & 1 \\
Dyspnoea & $3(3)$ & 0 & 0.24 \\
Bronchitis & $1(1)$ & $1(1)$ & 1 \\
Cerebrovascular accident and & $1(1)$ & 0 & 1 \\
$\quad$ death & & & \\
Haemoptysis & $1(1)$ & 0 & 1 \\
\hline
\end{tabular}

Data are presented as number of subjects (number of episodes) in each group, unless otherwise stated. COPD: chronic obstructive pulmonary disease. the presence of collateral ventilation have been debated [12, 21, $22]$, its presence being associated with safer outcomes and its absence most closely mimicking LVRS and the resulting efficacy by producing lobar atelectasis. The three mechanisms of improvement described for this therapy may have a combined effect, resulting in the improvement of these patients; however, in the absence of lobar atelectasis, redirection of airflow and a shift in lung volumes to less diseased areas of the lungs may be the prevailing mechanism. Other possible mechanisms to consider are a reduction in physiological dead space (leading to increased efficiency of ventilation), or a redirection of airflow to less diseased areas of the lung as a result of decreased distension at higher levels of ventilation (reduced dynamic hyperinflation).

In the absence of lobar atelectasis, the measured volumetric changes are small in comparison to the static measures of the entire lung; however, these volumes probably represent a large proportion of the tidal volume for each breath. Authors of a previous report postulated that small volumetric changes produced by valve treatments may have a significant impact because patients with advanced emphysema are not able to increase their tidal volume during activity [12].

Results of health status assessments showed improvements in SGRQ scores in treated and control subjects. The improvements in the control group probably reflected both placebo and study effects, and concur with results reported in the control groups of other blinded randomised studies [23-25]. Study effects can be expected as the subjects were part of a clinical trial with increased attention and monitoring of their disease (Hawthorne effect). In addition, the short period of blinding could have made the placebo effect more evident. This study also provides evidence of the profound effect of COPD exacerbations on health-related quality of life, as illustrated by worse total SGRQ scores in patients suffering exacerbations in both study groups. 
This study showed no effects in the majority of parameters used to evaluate clinical measures of disease severity, including pulmonary function tests and exercise capacity (table 4). Modest improvements in FEV1 following valve therapies have been shown in association with lobar atelectasis, but were not clinically significant in the entire study group [9, 10, 21]. However, other studies have shown that effort-dependent tests, such as FEV1, may not be the right parameters to evaluate improvement with this therapy in patients with advanced emphysema and no reversible airway disease [14, 26, 27]. With respect to exercise capacity, modest improvements have been shown with 6MWT results in association with atelectasis [10, 21]. HOPKINSON et al. [11] also showed improvements in exercise capacity (cycle endurance time) following valve therapy in the absence of atelectasis, which was linked to decreased dynamic hyperinflation during exercise or increased efficiency of breathing. The lack of improvements in 6MWT in our study may be explained because pulmonary rehabilitation was not required (influencing exercise results) and many subjects had end-stage disease and severe deconditioning. It is also possible that the $6 \mathrm{MWT}$ is rather insensitive to improvements confined to the lungs, since it may not improve with bronchodilator therapy. In this regard, endurance tests or evaluations of daily activity with accelerometers may have detected a benefit.

The device and procedure safety results were very encouraging in this study, and limited adverse events reported for the treated group compared favourably with the control group. The number of valves placed corresponded to the number of segmental airways in both upper lobes and there was no airway erosion, migration or expectoration of valves. In addition, nearly all subjects were discharged the day after the procedure without complications, and the presence of the valves did not seem to increase the risk of COPD exacerbations or pneumonia. These results are important because they show that this minimally invasive approach is safe and effective in this elderly and fragile population with emphysema.

This study has the following limitations. The measures of lobar volumes were done using automated software but, in some cases, the segmentation required visual editing by CT reviewers. The manual editing process was not blinded, as valves are visible on CT images; however, bias in this analysis was minimised by selecting an academic research laboratory with extensive experience in these evaluations and by using a coprimary end-point to determine responders. The blinded duration was short (as required by local ethics committees), and a longer duration may have elicited more easily interpretable results. For example, results of the 6-month evaluations indicate further improvements in the composite end-point. Placement of valves was directed by the protocol and not customised for each subject. It is possible that with improvements in understanding of this technique and CT analysis of lobar distributions of emphysema that treatment could be customised to specific subjects. Another limitation may be the small number and diverse group of subjects enrolled in this study. Finally, the impact that COPD exacerbation had on the short-term results and data analysis may have been another limiting factor, as subjects who remained exacerbation-free showed greater improvements than those who experienced an exacerbation.
In conclusion, this randomised, blinded and sham procedurecontrolled study of bronchial valve therapy demonstrated that the modality of treatment without complete lobar occlusion was safe but not effective in the majority of patients. In a minority of patients $(24 \%)$ the composite success criterion was achieved. There were no episodes of lobar atelectasis and/or pneumothorax, with a similarly low level of complications and adverse events in subjects treated with valves compared with the control group. These data suggest that additional studies are warranted to better identify the subgroup with a treatment response, to evaluate other bronchial valve treatment modalities and to improve patient selection criteria to achieve broader effectiveness.

\section{SUPPORT STATEMENT}

The funding for this study, including the supply of bronchial valves and funding for editorial support, was provided by Spiration Inc. (study number: CPR-01576).

\section{CLINICAL TRIAL}

This study is registered at Clinicaltrials.gov with identifier number NCT00880724.

\section{STATEMENT OF INTEREST}

Statements of interest for V. Ninane, L. Seijo, P.W. Jones, H.O. Coxson, S.C. Springmeyer and X. Gonzalez, and for the study itself can be found at www.erj.ersjournals.com/site/misc/statements.xhtml

\section{ACKNOWLEDGEMENTS}

The authors would like to thank A. Mugglin (Division of Biostatistics, University of Minnesota, Minneapolis, MN, USA) for his statistical support, and to acknowledge editorial support in the form of assistance in developing the manuscript first draft, assembling tables and figures, collating author comments and copyediting, which was provided by K. Hollingworth of Livewire Communications (Gerrards Cross, UK).

\section{REFERENCES}

1 World Health Organization. Chronic Obstructive Pulmonary Disease Fact Sheet. www.who.int/mediacentre/factsheets/fs315/ en/index.html Date last accessed: November 25, 2010. Date last updated: November 2010.

2 Halbert RJ, Natol JL, Gano A, et al. Global burden of COPD: systematic review and meta-analysis. Eur Respir J 2006; 28: 523-532.

3 Rodriguez-Roisin R, Anzueto A, Bourbeau J, et al. GOLD Executive Committee. Global Strategy for the Diagnosis, Management, and Prevention of Chronic Obstructive Pulmonary Disease. Global Initiative for Chronic Obstructive Lung Disease, 2011. Available from: http:/ / www.goldcopd.org/guidelines-global-strategy-fordiagnosis-management.html.

4 Celli BR, MacNee W, Agusti A, et al. Standards for the diagnosis and treatment of patients with COPD: a summary of the ATS/ERS position paper. Eur Respir J 2004; 23: 932-946.

5 Naunheim KS, Wood DE, Mohsenifar Z, et al. Long-term followup of patients receiving lung-volume-reduction surgery versus medical therapy for severe emphysema by the National Emphysema Treatment Trial Research Group. Ann Thorac Surg 2006; 82: 431-443.

6 Cooper JD, Patterson GA, Sundarexan RS, et al. Results of 150 consecutive bilateral lung volume reduction procedures in patients with severe emphysema. J Thorac Cardiovasc Surg 1996; 112: $1319-1330$

7 Berger RL, Wood KA, Cabral HJ, et al. Lung volume reduction surgery. A meta-analysis of randomized clinical trials. Treat Respir Med 2005; 4: 201-209. 
8 Naunheim KS. Lung-volume reduction surgery: a vanishing operation? J Thorac Cardiovasc Surg 2007; 133: 1412-1413.

9 Toma TP, Hopkinson NS, Hillier J, et al. Bronchoscopic volume reduction with valve implants in patients with severe emphysema. Lancet 2003; 361: 931-933.

10 Venuta F, di Giacomo T, Rendina EA, et al. Bronchoscopic lungvolume reduction with one-way valves in patients with heterogeneous emphysema. Ann Thoracic Surg 2005; 79: 411-416.

11 Hopkinson NS, Toma TP, Hansell DM, et al. Effect of bronchoscopic lung volume reduction on dynamic hyperinflation and exercise in emphysema. Am J Respir Crit Care Med 2005; 171: 453-460.

12 Springmeyer SC, Bollinger CT, Waddell TK, et al. Treatment of heterogeneous emphysema using the Spiration IBV Valves. Thorac Surg Clin 2009; 19: 247-253.

13 Hopkinson NS, Kemp SV, Toma TP, et al. Atelectasis and survival after bronchoscopic lung volume reduction for COPD. Eur Respir J 2011; 37: 1346-1351.

14 Coxson $\mathrm{HO}$, Nasute Fauerbach PV, Storness-Bliss C, et al. Computed tomography assessment of lung volume changes after bronchial valve treatment. Eur Respir J 2008; 32: 1443-1450.

15 Sterman DH, Mehta AC, Wood DE, et al. A multicenter pilot study of a bronchial valve for the treatment of severe emphysema. Respiration 2010; 79: 222-233.

16 Jones PW, Quirk FH, Baveystock CM. The St George's Respiratory Questionnaire. Respir Med 1991; 85: 25-31.

17 Jones PW. Interpreting thresholds for a clinically significant change in health status in asthma and COPD. Eur Respir J 2002; 19: 398-404.
18 Ware JE, Sherbourne CD. The MOS 36-item Short-Form health survey (SF-36): I. Conceptual framework and item selection. Med Care 1992; 30: 473-483.

19 Bestall JC, Paul EA, Garrod R, et al. Usefulness of the Medical Research Council (MRC) dyspnoea scale as a measure of disability in patients with chronic obstructive pulmonary disease. Thorax 1999; 54: 581-586.

20 Wood DE, McKenna RJ Jr, Yusen RD, et al. A multicenter trial of an intrabronchial valve for treatment of severe emphysema. J Thorac Cardiovasc Surg 2007; 133: 65-73.

21 Sciurba FC, Ernst A, Herth FJF, et al. A randomized study of endobronchial valves for advanced emphysema. $N$ Engl J Med 2010; 363: 1233-1244.

22 Noppen N. Collateral ventilation in end-stage emphysema: a blessing or a curse for new bronchoscopic treatment approaches (or both)? Respiration 2007; 74: 493-495.

23 Calverley P, Pauwels R, Vestbo J, et al. Combined salmeterol and fluticasone in the treatment of chronic obstructive pulmonary disease: a randomised controlled trial. Lancet 2003; 361: 449-456.

24 Tashkin DP, Celli B, Senn S, et al. A 4-year trial of tiotropium in chronic obstructive pulmonary disease. N Engl J Med 2008; 359: 1543-1554.

25 Calverley PM, Anderson JA, Celli B, et al. Salmeterol and fluticasone propionate and survival in chronic obstructive pulmonary disease. $N$ Engl J Med 2007; 356: 775-789.

26 Krowka MJ. Effect of effort on measurement of forced expiratory volume in one second. Am Rev Resp Dis 1987; 136: 829-833.

27 Reilly J. COPD and declining FEV1 - time to divide and conquer? $N$ Engl J Med 2008; 359: 1616-1618. 